\title{
FIRST SUPPLEMENT TO INSECTAE PORTORICENSIS
}

Because the question has been asked, and to avoid further uncertainty, it should here be definitely stated that, unless otherwise specified, the descriptions appearing in Insectae Portoricensis of new species are by the compiler of the list, George N. Wolcott.

P. 16.

Dr. J. W. Folsom has given the MS. name of Cremastocephalus bilobatus to the green Collembola commonly found on canna, water hyacinth, yautía, corn and sugar cane, discussed by Wolcott 21-10.

P. 20.

For Periplaneta australiae Fabricius, read Periplaneta australasiae Fabricius.

P. 32 .

Glyptotermes pubescens has been described by Dr. T. E. Snyder in No. 2496, Proc. U. S. National Museum, Vol. 64, Art. 6, pp. 1-49, pl. 1-5, 1924.

Nasutitermes (Tenuirostitermes) wolcotti has been described by Dr. Snyder in the Proceedings Entomological Society of Washington, Vol. 26, No. 5, May 1924, pp. 131-132.

P. 33.

For Pseudocaecillus, read Pseudocaecilius.

Mr. Rolla P. Currie has examined a small collection of Odonata from Porto Rico, and finds that the only species not recorded by Kolbe and Gundlach is Scapanea frontalis Burmeister.

He makes the following changes in the list of Odonata:

Erythragrion dominicanum Selys is now known as Telebasis dominicana Selys.

Erythragrion vulneratum Hagen is now known as Telebasis vulnerata Hagen.

For Aeschinidae, read Aeschnidae.

For Gymnacantha, read Gynacantha.

Libellula umbrata Linn. is now known as Erythrodiplax umbrata Iinn.

Orthemis discolor Burmeister is now known as Orthemis ferruginea Fabricius.

Dythemis discreta Hagen (which should have been spelled dicrota) is now known as Micrathyria didyma Selys. 
Diplax ambusta Hagen is now known as Erythrodiplax connata justiniana Selys.

Diplax portoricensis Kolbe (which should be portoricana) prob ably belongs in the genus Erythrodiplax, along with minuscula.

For D. miniscula, read D. minuscula.

$\mathrm{Mr}$. Currie has also bracketed the names of most of the authors, showing generic transfers, but for consistency with the rest of the list, these are not here indicated.

P. 42 .

Under Odyneurus dejectus Cresson, add

a large cluster on asparagus frond (224-23 det. Rohwer).

P. 43 .

Under Pseudagenia bella Cresson, add

reared from mud nests on the leaves of Inga vera at Cayey (366-22 det. Rohwer).

Under Crabro croesus Lepeltier, add

reared from cocoons in rotten log (78-23 det. Rohwer); on Mona Island (1308-13).

P. 44 .

Under Notogonidea vinulenta Cresson, add

on Mona Island (1310-13 det. Rohwer).

P. 60 .

For the author of Aspidiotiphagus citrinus, instead of Crawford, read Craw; Dr. L. O. Howard states "it was Alexander Craw who described this species."

P. 65 .

Add

Pimpla rufoniger Cresson - det. Cushman at Aibonito (SSC).

P. 80 .

For Omalodes kugii Marseul, read Omalodes krugii Marseul, add Carnicops dominicana ? Marseul - det. A. J. Mutchler under bark of Erythrina tree at Cayey (248-17).

P. 93.

Add

Stethorus punctum LeConte - det. Sicard (previously determined by Dr. Schwarz as "close to punctum Lec.")

on leaves of Psidium guajava and Spondias lutea (88-13, $722-16,838-16)$. 
P. 94.

After Psyllobora lineolate Fabricius, add — confirmed by Dr. Sicard

and delete "presumably this species" on the next line.

P. 113.

For Noda sp. or Nodonota sp. - det. Cotton, read

Nodonota wolcotti Bryant, G. E., in "New Species of Phytophaga" Annals and Magazine of Natural History, Ser. 9, Vol. 13, p 299, March, 1924, TYPE from Porto Rico.

P. 116.

For Homophoeta aequinoctailis Fabricius, read Homophoeta aequinoctialis Fabricius

P. 117.

Add

Hermaeophaga cubana Bryant, G. E., in Annals and Magazine ol: Natural History, Ser. 9, Vol. 13, p. 302, March, 1924, TYPE from Porto Rico.

millions on a few unidentified trees near Guayama (50-22).

P. 129 .

After the last record under Lachnopus coffeae montanus Mar shall, add

feeding on leaves of Cestrum macrophyllum Vent. (host de termination by R. A. Toro) at Ciales (34-24).

P. 209.

Add

Root, Francis Metcalf, "Notes on Mosquitoes and other BloodSucking Flies from Porto Rico." In American Journal of Hygiene, Vol. 2, No. 4, July 1922, pp. 394-405, figs. 5.

Notes on the following species:

CHIRONOMID卌.

Culicoides furens Poey (Syn. C. maculithorax Williston) SIMULIID ब.

Simulium quadrivittatum Loew

CULICIDA.

Anopheles albimanus Wiedemann

Anopheles grabhamii Theobald 
Uranotaenia socialis Theobald

Uranotaenia lowii Theobald

Aedes (Stegomyia) aegypti Linnaeus

Aedes (Taeniorhynchus) portoricensis Ludlow

Aedes (Ochlerotatus) ? condolescens Dyar \& Knab ? (larva described)

Psorophora jamaicensis Theobald

Culex (Culex) quinquefasciatus Say (Syn. C. fatigans Wied.)

Culex (Culex) nigripalpus Theobald, var. similis Theobald

Culex (Melanoconion) atratus Theobald (Syn. C. falsificator D. \& K.)

Culex (Choeroporpa) borinqueni sp. nov. Root

"The commonest 'wild' Culex of the Porto Rican costal plain. ... . . found breeding in all sorts of slow streams, pools and marshy places, . . . . at Río Piedras, Martin Peña and Aguirre."

Deinocerites cancer Theobald

TABANID

Chrysops costatus Fabricius - "mosca de manglar"

\section{MUSCID AE.}

Stomoxys calcitrans Linnaeus

P. 217.

For Conicera aldrichii Brues, read

Conicera latimana Malloch, J. R., "A New Species of Conicera from Porto Rico" in Proc. Ent. Soc. Washington, Vol. 26, No. 4, p. 73, April, 1924, TYPE from Porto Rico.

(as Conicera aldrichii Brues) Wetmore 16-74, eaten by hummingbird, Anthrocothorax aurulentus.

P. 222 .

To the rearing records of Nemorilla maculosa Macquart, add

from Tetralopha scabridella Ragonot at Cayey (385-22 det. Aldrich).

About the middle of the page, add

Phorocera parviteres Aldrich - det. Aldrich

from Pieris monuste Linn. at Yauco (77-23). ; (sp.) from Melanchroia cephise Cramer (6-24). 
P. 225 .

The rearing records for Sarcophaga robusta Aldrich are incorrect for this species. It should read

Sarcophaga robusta Aldrich

Aldrich 16-268: from Mayagüez, P. R.

Sarcophaga sternodontis Townsend

Aldrich 16-267: from Mayagüez, P. R.

Jones \& Wolcott 22-49: from pupae of Mocis (Remigia) repanda Fabr. and from white grubs. (452-12, 766-12, . . . etc.

P. 239.

Mr. A. C. Morgan of Clarksville, Tenn. has identified the following thrips (Thysanoptera) which are new to Porto Rico, and will describe the new genus and species in a forthcoming paper.

TEREHRANTIA.

Cercyothrips gen. et sp. nov.

collected by E. G. Smyth, October 7, 1919 at Río Piedras (685-19).

Limnothrips cerealium Haliday

on sugar-cane leaves at Guánica, March 18, 1920 (GNW).

Corynothrips stenopterus Williams

on "yuca", Agava sissalana, Nov. 18, 1919 at Río Piedras (788A-19).

Franklinothrips vespiformis Crawford

collected by E. G. Smyth at Río Piedras.

Heliothrips fasciatus Pergande

on alfalfa at Río Piedras, Nov. 16, 1923 (349-23), collected by F. Seín.

Anaphothrips sp. nov.

four females from leaves of sugar cane at Bayamón, May

$5,1920 \cdot($ GNW $)$.

Sericothrips sp. nov.

two females collected by E. G. Smyth at Río Piedras, March 25,1920 .

$$
\text { TUBULIFERA. }
$$

Ommatothrips gossypii Hood

on coffee leaves (GNW) ; on leaves of Inga vera at Cayey (306-23).

Gastrothrips sp. nov.

in stomach of lizard, Anolis cristatellus D. \& B., Oct. 3, 1923 (308-23). 
Diceratothrips sp. nov.

in rotten cotton boll injured by Pink Bollworm at Pt. Cangrejos (307-23); on leaves of Inga vera at Cayey (306-23).

Lissothrips subgen. et sp. nov.

from stomach of lizard, Anolis stratulus Cope, collected May 9, 1924, at Hacienda Santa Catalina, Mameyes by F. Seín.

Hindsiana cocois Watson

on leaves of sugar cane at Camuy, April 26, 1920 (GNW).

Hindsiana weigeli Watson

(probably from sugar cane) at Río Piedras, Feb. 23, 1920 (GNW).

P. 244 .

For Velidae, read Velidide.

P. 257.

For wild Bougainvillea vine, read Trichostigma octandra (L.) H. Walt (host determination by R. A. Toro).

P. 270 .

Add

Cubana tortriciformis Muir MS sp. nov.

from el Yunque (29-24).

P. 281.

To the host records of Pseudococcus bromeliae Bouché. add on aerial roots of "jagüey", Ficus laevigata, attended by "hormiguilla", at Manatí (24-24 det. Ferris).

Also add

Pseudococcus comstocki Kuwana - det. Ferris

from stomach of lizard, Anolis pulchellus D. \& B., at Toa Baja (lizard No. 306).

P. 291.

Add a host record for Pseudoparlatoria ostreata Cockerell, on Piper medium stems from Manatí (25-24 det. Ferris).

P. 306 .

Add

Opogona 206 